\title{
Attributable Risk Estimate of Severe Psoriasis on Major Cardiovascular Events
}

\author{
Nehal N. Mehta, MD, MSCE, FAHA ${ }^{a, b}$, YiDing Yu, BA ${ }^{a}$, Rebecca Pinnelas, BS ${ }^{a}$, Parasuram \\ Krishnamoorthy, MD ${ }^{\mathrm{a}}$, Daniel B. Shin, BA ${ }^{\mathrm{c}}$, Andrea B. Troxel, ScD ${ }^{\mathrm{c}, \mathrm{d}}$, and Joel M. Gelfand, \\ MD, MSCE ${ }^{\mathrm{b}, \mathrm{c}, \mathrm{d}}$ \\ aPreventive Cardiology, Inflammatory Risk, Department of Medicine, University of Pennsylvania \\ School of Medicine \\ ${ }^{b}$ Center for Clinical Epidemiology and Biostatistics, University of Pennsylvania School of Medicine \\ 'Department of Dermatology, University of Pennsylvania School of Medicine \\ ${ }^{d}$ Department of Epidemiology and Biostatistics at the University of Pennsylvania School of \\ Medicine
}

\begin{abstract}
Background-Recent studies suggest that psoriasis, particularly if severe, may be a risk factor for major adverse cardiac events such as myocardial infarction, stroke, and mortality from cardiovascular disease. We compared the risk of major adverse cardiac events between patients with psoriasis and the general population and estimated the attributable risk of severe psoriasis.

Methods-We performed a cohort study in the General Practice Research Database. Severe psoriasis was defined as receiving a psoriasis diagnosis and systemic therapy $(\mathrm{N}=3,603)$. Up to 4 patients without psoriasis were selected from the same practices and start dates for each patient with psoriasis $(\mathrm{N}=14,330)$.
\end{abstract}

Results-Severe psoriasis was a risk factor for major adverse cardiac events (hazard ratio 1.53; $95 \%$ confidence interval $1.26,1.85$ ) after adjusting for age, gender, diabetes, hypertension, tobacco use and hyperlipidemia. After fully adjusted analysis, severe psoriasis conferred an additional $6.2 \%$ absolute risk of 10-year major adverse cardiac events.

Conclusions-Severe psoriasis confers an additional 6.2\% absolute risk of 10-year rate of major adverse cardiac events compared to the general population. This potentially has important therapeutic implications for cardiovascular risk stratification and prevention in patients with severe psoriasis. Future prospective studies are needed to validate these findings.

\section{Keywords}

Psoriasis; major adverse cardiac events; inflammation; risk factors

\footnotetext{
Corresponding author: Nehal N. Mehta, MD, MSCE, FAHA, 6 Penn Tower, 3400 Civic Center Boulevard, Philadelphia, PA 19104, Phone: 215.662.7988; Fax: 215.662.7919; Pager: 215.308.6762, Nehal.Mehta@ uphs.upenn.edu.

Potential Conflicts of Interest: Dr. Gelfand has grants from Amgen, Pfizer and Abbott, and is a consultant for Amgen, Abbott, Celgene and Centocor. The other authors confirm that there are no other potential conflicts of interest.

Authorship Verification: All authors had access to the data and meet requirements for authorship for this manuscript.

Financial Disclosure: The funders had no role in study design, data collection and analysis, decision to publish, or preparation of the manuscript.
} 


\section{Introduction}

Psoriasis is a common, chronic inflammatory disease of the skin and joints that affects about $2-4 \%$ of the general population. Psoriasis patients report impaired quality of life ${ }^{1}$, even in mild cases, and severely affected psoriasis patients have a 5-year shorter life expectancy, mostly due to cardiovascular disease. ${ }^{2}$ Patients with psoriasis have increased prevalence of traditional cardiovascular risk factors such as diabetes, hypertension, dyslipidemia, tobacco use, and obesity. ${ }^{3}$ Importantly, even after adjusting for these risk factors, psoriasis has been shown to be a risk factor for atherosclerosis, coronary artery disease, endothelial dysfunction, myocardial infarction, stroke, and cardiovascular mortality. ${ }^{4-8}$ Shared inflammatory pathways common to psoriasis and atherosclerosis are thought to play an important role in this interaction, but the exact mechanisms are still unknown. ${ }^{9}, 10$

Recognizing the increased prevalence of cardiovascular disease in persons with psoriasis, consensus statements have been published recognizing psoriasis as an emerging cardiac risk factor. ${ }^{11,12}$ Yet despite numerous studies linking psoriasis and cardiovascular disease, translating these studies into clinical guidelines has been hampered by the lack of accurate cardiovascular risk prediction in the psoriasis population.

The importance of cardiovascular risk prediction is illustrated by the Adult Treatment Panel (ATP) III, which guides management of lipoprotein abnormalities in the United States. ${ }^{13}$ The philosophy of the ATP III is that the intensity of treatment and lifestyle modification should match the level of absolute predicted cardiovascular risk as assessed by the Framingham Risk Score (FRS). The FRS assigns weighted points to traditional risk factors (age, sex, hyperlipidemia, hypertension, blood pressure, diabetes, and smoking status) in order to predict major adverse cardiac events ${ }^{14}$ and coronary heart disease.${ }^{15}$ However, the ATP III also recognizes that there are emerging risk factors not captured in the FRS which "contribute to [coronary heart disease] risk to varying degrees," "can have utility in selected persons to guide intensity of risk reduction therapy," and whose presence can "modulate clinical judgment when making therapeutic decisions". ${ }^{13}$

Therefore, an important question facing providers caring for patients with psoriasis is how psoriasis should impact intensity of risk reduction therapy as an emerging cardiac risk factor. Because any additional intensity of therapy should match the magnitude of additional risk, an estimate of the attributable risk of psoriasis on major adverse cardiac events is needed to guide accurate risk stratification and risk reduction therapy in the clinical setting. Therefore, the objectives of the present study were twofold: first, to compare the incidence of major adverse cardiac events in severe psoriasis and the general population in a large observational study and, second, to quantify the attributable risk of psoriasis on major adverse cardiac events in order to understand how to best risk stratify patients for cardiovascular prevention.

\section{Methods}

\section{Study population and data source}

The study was conducted and is reported based on recommendations of the STROBE statement. ${ }^{16}$ The study population was derived from the General Practice Research Database (GPRD), a medical records database in the United Kingdom (UK) that was established for epidemiologic research in 1987. Details of the GPRD, including validation of psoriasis, cardiovascular risk factors and cardiovascular disease, have been published elsewhere. 3, 7, 17 


\section{Time period and age eligibility}

Data were obtained between 1987-2002 for patients who were 18 years or older at the date their person-time began. For the primary analysis, patients with a history of cardiovascular disease, defined as a code of ischemic heart disease, myocardial infarction, transient ischemic attack, stroke or peripheral arterial disease on or before the start date, were excluded.

\section{Definition of exposure}

We defined patients with severe psoriasis as those with a diagnostic code of psoriasis and history of systemic therapy consistent with severe psoriasis, including phototherapy, PUVA, methotrexate, azathioprine, cyclosporine, oral retinoids (etretinate, acitretin), hydroxyurea, and mycophenolate mofetil. Of note, during the time period in which this study was conducted, biologic therapies were not approved for treatment of psoriasis in the UK and therefore are not represented in this dataset. The unexposed population (controls) was composed of patients with no history of a psoriasis diagnostic code.

\section{Sampling of exposed and unexposed cohorts}

All patients with severe psoriasis (as defined above) who were 18 years or older at their index date, and had at least 1 day of observation time, were included. For patients with severe psoriasis, their index date was the first date on or after the first diagnosis of psoriasis in which the patient received a code for treatment consistent with severe disease. For patients without psoriasis, their index date was the date of a medical record entry which was within 60 days of the psoriasis index date. Up to 4 unexposed subjects who were 18 years or older at their index date were randomly selected for each patient with psoriasis, matched on practice, date of registration in the practice, and psoriasis index date (corresponding to a medical record date of the unexposed patient).

\section{Person-time calculation}

For patients with severe psoriasis, follow-up started at the latest date when the patient could first be defined as having severe psoriasis (e.g. received a treatment code consistent with severe disease), was registered with the practice, or the practice was deemed up to standard (UTS), defined as having sufficient data meeting audit requirements to participate in research. For unexposed subjects, follow-up started at the latest date when the patient was registered in the practice, the index date (matched to the psoriasis index date), and the date that the practice was deemed UTS. For all groups, follow-up ended at the date of death, development of an outcome of interest, transfer out of the practice, or end of UTS.

\section{Outcome of interest}

The outcome of interest was first recorded major adverse cardiac event, defined as occurrence of non-fatal myocardial infarction, non-fatal stroke, or death due to cardiovascular etiology. Outcomes were determined by diagnostic codes consistent with acute myocardial infarction, acute ischemic stroke, or mortality secondary to myocardial infarction, stroke, or arrhythmia. These outcomes have been previously validated for research in the GPRD ${ }^{17-19}$ and have been demonstrated by our group and others. ${ }^{4,7,20-22}$

\section{Co-variables of interest}

We identified traditional cardiovascular risk factors including age, sex, hypertension, diabetes, hyperlipidemia, and smoking (current, former, never) by the presence of diagnostic codes. BMI was extracted from patient charts in $69 \%$ of the cohort. 


\section{Statistical Analysis}

The sample size was determined by including the maximum eligible number of patients with severe psoriasis based on age criteria. We randomly selected up to 4 unexposed subjects per patient with psoriasis, as additional matching yields minimal increases in statistical power. Data were summarized descriptively and differences in dichotomous and continuous variables were examined between patients with and without psoriasis. Dichotomous variables were tested with Fisher's exact test. Continuous variables were tested with a T-test if they were normally distributed, or with the Wilcoxon test if the data were not normally distributed. We fit age- and sex-adjusted Cox proportional hazards models to determine the overall hazard ratio (HR) of major adverse cardiac events comparing psoriasis patients to those without psoriasis. The primary model included major cardiovascular risk factors. Multiple sensitivity analyses were performed to test the underlying assumptions of our primary analysis and to test the robustness of our findings. All analyses were performed using STATA 10.0, and a two-sided p-value $<0.05$ was the threshold for statistical significance.

We applied the adjusted HR of psoriasis obtained from the primary model to the following formula to estimate 10-year incidence of major adverse cardiac events: adjusted HR for major adverse cardiac events in psoriasis $\times$ baseline incidence of major adverse cardiac events in the unexposed population resulted in an adjusted incidence rate (IR) for major adverse cardiac events in the psoriasis group. We subtracted the baseline incidence of major adverse cardiac events in the unexposed population from this adjusted IR of major adverse cardiac events in psoriasis to obtain the excess attributable risk of psoriasis. The attributable risk of psoriasis was multiplied by ten to obtain 10-year risk of major adverse cardiac events due to psoriasis.

\section{Protection of Human Subjects}

This study was approved by the University of Pennsylvania Institutional Review Board and by the Independent Scientific Advisory Committee of the Medicines and Healthcare Products Regulatory Agency of the United Kingdom Department of Health. The study was conducted in accordance with the Declaration of Helsinki.

\section{Results}

We identified 3,603 patients with severe psoriasis and 14,330 matched unexposed patients (Table 1). Patients with severe psoriasis were slightly older, more likely to be male, and more likely to have established cardiovascular risk factors than patients without psoriasis. Therapies for patients with severe psoriasis were consistent with patterns of use prior to approval of biologic therapies and included methotrexate (58.7\%), phototherapy (16.9\%), azathioprine (16.2\%), cyclosporine (10.8\%), oral retinoids (9.2\%), hydroxyurea (5.8\%), and mycophenolate mofetil (0.3\%).

\section{Psoriasis is associated with increased risk of major adverse cardiac events}

The incidence of major adverse cardiac events in unexposed patients and psoriasis patients is shown in Table 2. The frequency of major adverse cardiac events was higher in patients with severe psoriasis compared to the unexposed group $(4.5 \%$ vs $2.9 \%, \mathrm{p}<0.001)$. The unadjusted overall risk of major adverse cardiac events per 1,000 person-years was significantly increased in patients with severe psoriasis $(16.4,95 \%$ confidence interval 14.3, 18.9) compared to unexposed patients $(11.6,95 \%$ CI 10.7, 12.6). The unadjusted HR for major adverse cardiac events was 1.43 (95\% CI 1.22, 1.67). 
After adjusting for traditional cardiovascular risk factors (age, sex, hyperlipidemia, hypertension, smoking, diabetes), severe psoriasis remained a risk factor for major adverse cardiac events (HR 1.53; 95\% CI 1.26, 1.85). Analysis by gender did not reveal significant differences. Finally, the results were robust to a variety of sensitivity analyses shown in Table 3, which were important to assess prior to utilizing the adjusted HR to estimate the impact of psoriasis on 10-year major adverse cardiac events. Obesity was not found to be a significant confounder when reported by the general practitioner, but was missing in approximately $31 \%$ of patients and was therefore not included in the primary analysis.

\section{Psoriasis confers an attributable risk of $6.2 \%$ on 10 -year incidence of major adverse cardiac events}

After severe psoriasis was determined to be a risk factor for major adverse cardiac events through Cox hazard modeling, we quantified the yearly attributable risk of psoriasis on major adverse cardiac events to understand the long-term impact of psoriasis on cardiovascular disease. The adjusted HR for major adverse cardiac events in psoriasis from the primary model was 1.531297 (Table 3). This number was multiplied by the baseline incidence rate of major adverse cardiac events in the general population from the GPRD ( 0.0115902 events per year). This yielded the adjusted incidence rate for the exposed group (0.017748). The baseline rate (0.0115902 major adverse cardiac events per year) was then subtracted from the adjusted incidence rate $(0.017748)$, which yielded the excess rate of major adverse cardiac events per year (0.006158) within the severe psoriasis group. Assuming the excess yearly risk is constant over time, this results in an estimated 10-year risk of major adverse cardiac events of approximately $6.2 \%$.

\section{Discussion}

The primary objective of this cohort study was to examine the incidence of major adverse cardiac events, a metric of the FRS for cardiovascular risk assessment ${ }^{14}$, in patients with severe psoriasis. We found that patients with severe psoriasis had increased incidence of major adverse cardiac events after controlling for traditional cardiovascular risk factors. The second objective was to estimate the excess absolute risk that psoriasis confers on 10-year major adverse cardiac events, which we found to be $6.2 \%$. To our knowledge, this study is the first to estimate the magnitude of cardiovascular risk attributable to severe psoriasis to better risk stratify patients for preventive cardiovascular care.

Consistent with prior work from our group ${ }^{4,7,22}$ and others ${ }^{6,23}$, severe psoriasis was a risk factor for major adverse cardiac events in this cohort study after adjustment for major cardiovascular risk factors. Given the heterogeneous nature of clinical atherosclerosis, major adverse cardiac events has served as the endpoint for large cardiovascular trials and risk assessment tools such as the FRS. ${ }^{14}$ The FRS is the main tool utilized by practitioners for risk stratification of cardiovascular disease within the United States. Based on the percent risk of 10-year major adverse cardiac events using FRS, practitioners determine whether patients are at low, moderate, or high risk for cardiovascular events and manage lipid, blood pressure, and blood glucose goals accordingly. Emerging risk factors that increase the risk of 10 -year major adverse cardiac events may therefore reclassify patients to higher risk categories and warrant more aggressive clinical management.

The attributable risk of severe psoriasis on major adverse cardiac events may therefore have significant clinical implications. We quantified the magnitude of 10 -year risk conferred by severe psoriasis by utilizing the baseline incidence rate of major adverse cardiac events in the general population and the adjusted HR of psoriasis. The absolute percentage impact of $6.2 \%$ is substantial, and on the population level can inform and guide treatment initiation and goals of risk reduction therapy. For example, both the ATP III for lipoprotein therapy 
and the Joint National Committee (JNC) 7 for blood pressure management use coronary heart disease risk stratification to guide lifestyle and medical therapy. While our attributable risk estimate of $6.2 \%$ includes the excess risk of stroke in addition to coronary heart disease, it provides important information regarding a patient's excess risk for global cardiovascular disease. This may assist clinicians considering cardiovascular risk reduction strategies for psoriasis patients who may be at the upper margin of their risk category and who may therefore benefit from more aggressive risk reduction therapy.

\section{Study Strengths and Limitations}

The use of the GPRD is a major strength of this study because it broadly represents the UK population, captures high quality, real world data on diseases, treatments, and outcomes, and has been validated in cardiovascular and psoriasis studies. ${ }^{24}$ However, there are important limitations to consider while interpreting this study. First, because data to construct continuous variables for hypertension and hyperlipidemia were not available, we generated dichotomous variables from diagnostic codes previously validated for FRS. While we adjusted for age, gender, tobacco use, diabetes, hypertension, and hyperlipidemia in our models, the lack of laboratory data hindered our ability to directly estimate the FRS for our cohorts, and therefore we could not directly assess whether FRS underestimates cardiovascular risk in patients with psoriasis. In addition, other biomarkers of interest, such as $\mathrm{C}$ reactive protein, could not be assessed in our study. Second, our definition of exposure requires a diagnostic code for psoriasis and systemic therapy consistent with severe psoriasis, but we are unable to discriminate between systemic therapy prescribed for psoriasis or other comorbidity, leaving the potential for misclassification of our exposure. Nevertheless, our sensitivity analyses indicate that this source of error is unlikely to impact our results. Moreover, the most commonly used therapy for severe psoriasis in this study was methotrexate, which several studies suggest is cardioprotective. ${ }^{25}$ Thus, we may be underestimating the risk of major adverse cardiac events in patients with severe psoriasis who are not concomitantly treated with methotrexate. Additionally, only severe psoriasis, and not mild disease, could be considered in this analysis as data about cardiovascular mortality in patients with mild psoriasis are not available. Previous work by our group and others suggests that the excess risk of cardiovascular events in patients with mild psoriasis is modest and therefore 10-year risk of major adverse cardiac events attributed to mild psoriasis is anticipated to be small and unlikely to meaningfully impact 10-year risk estimates. ${ }^{4,26,27}$ In addition, there is the potential for misclassification of cardiovascular risk factors used in our multivariate analyses that could potentially influence our ability to adjust for confounding. We note however that our methods for defining cardiovascular risk factors such as hypertension, hyperlipidemia, diabetes mellitus, and tobacco use have been well-validated by previously published literature and are based on diagnostic codes and prescription drug records from each patient's general practitioner. ${ }^{17-21}$ Nonetheless, there may be unmeasured or incompletely measured confounding variables in our data, and therefore affect our estimate of the attributable risk of severe psoriasis on major adverse cardiac events. It is also important to note that our estimate is averaged over all age groups and we expect this to have variation with respect to baseline risk. Because major adverse cardiac events outcomes are rare, even larger studies are needed to provide stability to estimates for young, low-risk groups.

Ultimately, GPRD reflects data available to general practitioners and offers a real-world example of how psoriasis may be incorporated into existing cardiovascular risk stratification strategies. While a prospective cohort study is the gold standard for estimating attributable risk, such a study poses significant cost and logistical challenges and is not presently available to our knowledge. ${ }^{28}$ Our cohort study represents a strong alternative. Our findings suggest that severe psoriasis is an important predictor of major adverse cardiac events and 
this information should guide clinicians seeking to manage psoriasis as an emerging cardiac risk factor. Future research should attempt to apply this method for estimating additional risk to a real-world cohort of patients with psoriasis and assess the predictive power of FRS in psoriasis patients compared to the general population. In addition, further studies are needed to evaluate the clinical utility of psoriasis in cardiovascular risk prediction and investigate the impact of psoriasis treatment on cardiovascular outcomes.

\section{Acknowledgments}

Funding Sources: This work was supported by a National Psoriasis Foundation Award (NNM), NHLBI K23HL97151 (NNM), a grant from the Doris Duke Charitable Foundation (YY), grant RO1HL089744 from NHLBI (JMG), and the Psoriasis Research Foundation in Honor of Herman Beerman (JMG).

\section{References}

1. Krueger G, Koo J, Lebwohl M, et al. The impact of psoriasis on quality of life: results of a 1998 National Psoriasis Foundation patient-membership survey. Arch Dermatol. 2001; 137:280-284. [PubMed: 11255325]

2. Abuabara K, Azfar RS, Shin DB, et al. Cause-specific mortality in patients with severe psoriasis: a population-based cohort study in the U.K. Br J Dermatol. 2010; 163:586-592. [PubMed: 20633008]

3. Neimann AL, Shin DB, Wang X, et al. Prevalence of cardiovascular risk factors in patients with psoriasis. J Am Acad Dermatol. 2006; 55:829-835. [PubMed: 17052489]

4. Gelfand JM, Neimann AL, Shin DB, et al. Risk of myocardial infarction in patients with psoriasis. JAMA. 2006; 296:1735-1741. [PubMed: 17032986]

5. Ludwig RJ, Herzog C, Rostock A, et al. Psoriasis: a possible risk factor for development of coronary artery calcification. Br J Dermatol. 2007; 156:271-276. [PubMed: 17223866]

6. Prodanovich S, Kirsner RS, Kravetz JD, et al. Association of psoriasis with coronary artery, cerebrovascular, and peripheral vascular diseases and mortality. Arch Dermatol. 2009; 145:700703. [PubMed: 19528427]

7. Mehta NN, Azfar RS, Shin DB, et al. Patients with severe psoriasis are at increased risk of cardiovascular mortality: cohort study using the General Practice Research Database. Eur Heart J. 2010; 31:1000-1006. [PubMed: 20037179]

8. Balci DD, Balci A, Karazincir S, et al. Increased carotid artery intima-media thickness and impaired endothelial function in psoriasis. J Eur Acad Dermatol Venereol. 2009; 23:1-6. [PubMed: 18702627]

9. Davidovici BB, Sattar N, Jorg PC, et al. Psoriasis and Systemic Inflammatory Diseases: Potential Mechanistic Links between Skin Disease and Co-Morbid Conditions. J Invest Dermatol. 2010; 130:1785-1796. [PubMed: 20445552]

10. Alexandroff AB, Pauriah M, Camp RD, et al. More than skin deep: atherosclerosis as a systemic manifestation of psoriasis. Br J Dermatol. 2009; 161:1-7. [PubMed: 19500102]

11. Kimball AB, Gladman D, Gelfand JM, et al. National Psoriasis Foundation clinical consensus on psoriasis comorbidities and recommendations for screening. J Am Acad Dermatol. 2008; 58:10311042. [PubMed: 18313171]

12. Friedewald VE, Cather JC, Gelfand JM, et al. AJC editor's consensus: psoriasis and coronary artery disease. Am J Cardiol. 2008; 102:1631-1643. [PubMed: 19064017]

13. Executive Summary of The Third Report of The National Cholesterol Education Program (NCEP). Expert Panel on Detection, Evaluation, And Treatment of High Blood Cholesterol In Adults (Adult Treatment Panel III). JAMA. 2001; 285:2486-2497. [PubMed: 11368702]

14. D'Agostino RB Sr, Vasan RS, Pencina MJ, et al. General cardiovascular risk profile for use in primary care: the Framingham Heart Study. Circulation. 2008; 117:743-753. [PubMed: 18212285]

15. Wilson PW, D'Agostino RB, Levy D, et al. Prediction of coronary heart disease using risk factor categories. Circulation. 1998; 97:1837-1847. [PubMed: 9603539] 
16. von Elm E, Altman DG, Egger M, et al. The Strengthening the Reporting of Observational Studies in Epidemiology (STROBE) statement: guidelines for reporting observational studies. Lancet. 2007; 370:1453-1457. [PubMed: 18064739]

17. Jick H, Jick SS, Derby LE. Validation of information recorded on general practitioner based computerised data resource in the United Kingdom. BMJ. 1991; 302:766-768. [PubMed: 2021768]

18. Khan NF, Harrison SE, Rose PW. Validity of diagnostic coding within the General Practice Research Database: a systematic review. Br J Gen Pract. 2010; 60:e128-e136. [PubMed: 20202356]

19. Hammad TA, McAdams MA, Feight A, et al. Determining the predictive value of Read/OXMIS codes to identify incident acute myocardial infarction in the General Practice Research Database. Pharmacoepidemiol Drug Saf. 2008; 17:1197-1201. [PubMed: 18985705]

20. Gibbs RG, Newson R, Lawrenson R, et al. Diagnosis and initial management of stroke and transient ischemic attack across UK health regions from 1992 to 1996: experience of a national primary care database. Stroke. 2001; 32:1085-1090. [PubMed: 11340214]

21. Nightingale AL, Farmer RD. Ischemic stroke in young women: a nested case-control study using the UK General Practice Research Database. Stroke. 2004; 35:1574-1578. [PubMed: 15143296]

22. Gelfand JM, Dommasch ED, Shin DB, et al. The risk of stroke in patients with psoriasis. J. Invest Dermatol. 2009; 129:2411-2418. [PubMed: 19458634]

23. Ahlehoff O, Gislason GH, Charlot M, et al. Psoriasis is associated with clinically significant cardiovascular risk: a Danish nationwide cohort study. J Intern Med. 2010

24. Gelfand JM, Weinstein R, Porter SB, et al. Prevalence and treatment of psoriasis in the United Kingdom: a population-based study. Arch Dermatol. 2005; 141:1537-1541. [PubMed: 16365254]

25. Prodanowich S, Ma F, Taylor JR, et al. Methotrexate reduces incidence of vascular diseases in veterans with psoriasis or rheumatoid arthritis. J Am Acad Dermatol. 2005; 52:262-267. [PubMed: 15692471]

26. Gelfand JM, Azfar RS, Shin DB, et al. Incidence of stroke in patients with psoriasis: A populationbased study. J Investigative Dermatol. 2008; 128:S81.

27. Brauchli YB, Jick SS, Miret M, Meier CR. Psoriasis and risk of incident myocardial infarction, stroke or transient ischaemic attack: an inception cohort study with a nested case-control analysis. Br J Dermatol. 2009; 160:1048-1056. [PubMed: 19210501]

28. Downs JR, Clearfield M, Weis S, et al. Primary prevention of acute coronary events with lovastatin in men and women with average cholesterol levels: results of AFCAPS/TexCAPS. Air Force/ Texas Coronary Atherosclerosis Prevention Study. JAMA. 1998; 279:1615-1622. [PubMed: 9613910] 
Table 1

Characteristics of Study Group

\begin{tabular}{|c|c|c|c|}
\hline Characteristics & $\begin{array}{l}\text { Unexposed } \\
(n=14,330)\end{array}$ & $\begin{array}{l}\text { Psoriasis } \\
(\mathbf{n}=\mathbf{3 , 6 0 3})\end{array}$ & P-value $^{\wedge}$ \\
\hline Male & $5,783(40.4 \%)$ & $1,750(48.6 \%)$ & $\mathrm{p}<0.01$ \\
\hline $\begin{array}{l}\text { Age, } \mathrm{y}^{*} \\
\text { Mean } \pm \mathrm{SD}^{*}\end{array}$ & $49.7 \pm 19.3$ & $52.2 \pm 16.7$ & $\mathrm{p}<0.01$ \\
\hline Diabetes mellitus & $737(5.1 \%)$ & $270(7.5 \%)$ & $\mathrm{p}<0.01$ \\
\hline History of myocardial infarction & $375(2.6 \%)$ & $116(3.2 \%)$ & $\mathrm{p}=0.05$ \\
\hline History of stroke & $268(1.9 \%)$ & $89(2.5 \%)$ & $\mathrm{p}=0.02$ \\
\hline History of TIA & $243(1.7 \%)$ & $68(1.9 \%)$ & $\mathrm{p}=0.43$ \\
\hline Hyperlipidemia & $842(5.9 \%)$ & $250(6.9 \%)$ & $\mathrm{p}=0.02$ \\
\hline Hypertension & $3,049(21.3 \%)$ & $858(23.8 \%)$ & $\mathrm{P}<0.01$ \\
\hline \multicolumn{4}{|l|}{ Smoking } \\
\hline None & $10,465(73.0 \%)$ & $2,488(69.1 \%)$ & $\mathrm{p}<0.01$ \\
\hline Current & $755(5.3 \%)$ & $241(6.7 \%)$ & \\
\hline Former & $3,110(21.7 \%)$ & $874(24.3 \%)$ & \\
\hline \multicolumn{4}{|l|}{$\mathrm{BMI}^{\dagger}$} \\
\hline$<25$ & $5,057(51.2 \%)$ & $1,025(42.1 \%)$ & $\mathrm{p}<0.01$ \\
\hline$\geq 25 \&<30$ & $3,291(33.3 \%)$ & $860(35.4 \%)$ & \\
\hline$\geq 30$ & $1,522(15.4 \%)$ & $548(22.5 \%)$ & \\
\hline \multicolumn{4}{|l|}{ Reason for end of study } \\
\hline Death & $790(5.5 \%)$ & $297(8.2 \%)$ & $\mathrm{p}<0.01$ \\
\hline End of UTS & $11,247(78.5 \%)$ & $2,860(79.4 \%)$ & \\
\hline Transfer out & $2,293(16.0 \%)$ & $446(12.4 \%)$ & \\
\hline
\end{tabular}

Derived using t-test except where $*$ denotes use of Wilcoxon test.

${ }^{\dagger}$ Data for BMI were available for $69 \%$ of the patients.

*Abbreviations: BMI, body mass index (calculated as weight in kilograms divided by height in meters squared); IQR, interquartile range; SD, standard deviation; TIA, transient ischemic attack; UTS, up to standard.

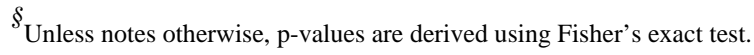


Table 2

Incidence of Major Adverse Cardiac Events in Patients with Psoriasis Compared with Patients without Psoriasis (Unexposed population)

\begin{tabular}{|l|c|c}
\hline Variable & Unexposed & Psoriasis \\
\hline \begin{tabular}{l|c|c} 
Follow-up time, y \\
Mean \pm SD
\end{tabular} & $3.4 \pm 2.8$ & $3.4 \pm 2.7$ \\
\hline Number of person-years & $48,661.8$ & $12,346.3$ \\
\hline Number of major adverse cardiac events $(\%)$ & $148(2.90)$ & $384(4.50)$ \\
\hline Incidence per 1,000 person-years $(95 \% \mathrm{CI})$ & $11.6(10.7,12.6)$ & $16.4(14.3,18.9)$ \\
\hline
\end{tabular}

${ }^{\dagger}$ Abbreviations: CI, confidence interval; IQR, interquartile range; SD, standard deviation. 
Table 3

Sensitivity Analysis Hazard Ratio Point Estimates

\begin{tabular}{|l|c|c|c|}
\hline Covariate & $\begin{array}{c}\mathbf{N} \\
\text { Unexposed }\end{array}$ & $\begin{array}{c}\mathbf{N} \\
\text { Psoriasis }\end{array}$ & $\begin{array}{c}\text { Model Hazard } \\
\text { Ratio (95\% CI) }\end{array}$ \\
\hline Primary analysis & 14,330 & 3,603 & $1.53(1.26,1.85)$ \\
\hline $\begin{array}{l}\text { Inclusion of patients with at least 1 GP } \\
\text { visit per year on average }\end{array}$ & 13,643 & 3,563 & $1.50(1.23,1.81)$ \\
\hline $\begin{array}{l}\text { Primary model with exclusion of } \\
\text { methotrexate }\end{array}$ & 13,289 & 1,358 & $1.86(1.44,2.41)$ \\
\hline $\begin{array}{l}\text { Primary model with exclusion of oral } \\
\text { retinoids or cyclosporine }\end{array}$ & 13,253 & 2,653 & $1.42(1.14,1.77)$ \\
\hline $\begin{array}{l}\text { Primary model restricted to patients } \\
\text { who received oral retinoids }\end{array}$ & 13,253 & 303 & $1.56(1.05,2.32)$ \\
\hline $\begin{array}{l}\text { Primary model with exclusion of } \\
\text { psoriatic arthritis }\end{array}$ & 13,289 & 1,156 & $1.44(1.16,1.78)$ \\
\hline $\begin{array}{l}\text { Inclusion of patients with at least 6 } \\
\text { months of person time }\end{array}$ & 11,832 & 2,963 & $1.60(1.32,1.95)$ \\
\hline Primary model with BMI included* & 9,870 & 2,433 & $1.71(1.32,2.18)$ \\
\hline $\begin{array}{l}\text { Primary model without BMI included } \\
\text { in those who had BMI measured* }\end{array}$ & 9,870 & 2,433 & $1.70(1.32,2.17)$ \\
\hline
\end{tabular}

BMI is included in $n=12,303$ or $69 \%$ of patients.

Abbreviations: BMI, body mass index; CI, confidence interval; GP, general practitioner; TIA, transient ischemic attack. 